\title{
Time Series Analysis in Modeling of Hepatitis B Incidence
}

\author{
Hua ZHANG ${ }^{1,2}$, Mingdong HUO ${ }^{3}$, Yao TONG ${ }^{1}$, *Pei LIU ${ }^{2,4}$ \\ 1. Department of Medical Insurance, School of Public Health, Southeast University, Nanjing, Jiangsu, China \\ 2. Key Laboratory of Environmental Medicine Engineering, Ministry of Education, School of Public Health, Southeast University, \\ Nanjing, China \\ 3. Zhongda Hospital Affiliated to Southeast University, Nanjing, Jiangsu, China \\ 4. Department of Epidemiology and Biostatistics, School of Public Health, Southeast University, Nanjing, Jiangsu, China
}

*Corresponding Author: Email: liupeiseu@163.com

(Received 10 Jan 2018; accepted 28 Jan 2018)

\section{Dear Editor-in-Chief}

With up to 240 million people chronically infected with hepatitis B worldwide (1), Hepatitis B is considered to be one of the most serious infectious diseases. "China was a high endemic area of hepatitis B virus infection, around 100 million people (about $10 \%$ of the Chinese population) are chronically infected" (2). In the study of infectious diseases, using time series to construct related prediction model has been very common and valuable $(3,4)$.

In this study, time series analysis was carried out to explore the epidemic trend and improve the prediction accuracy of hepatitis B in Jiangsu Province. Incidence data from 1990 to 2013 came from the Infectious Diseases Information System, SPSS 19.0 (Chicago, IL, USA) was used for statistical analysis. The study will provide basis for policy making of hepatitis B control.

From the following figure and run test, we can see that the sequence was non-stationary. Through the logarithmic conversion and secondorder non-seasonal differences, it was made smooth. The white noise test was done by referring to the residual value of the fitting data, and the accuracy of the model was investigated.

The incidence of hepatitis B in Jiangsu Province from 1990 to 2013 had a trend of change. The ARIMA $[0,2,1]$ model was constructed and fit well. The correlation function was in $95 \%$ confidence interval. The dynamic trend trajectories of fitting values of ARIMA [0, 2, 1] model are basically consistent with the trajectories of the actual values (Figs. 1,2).

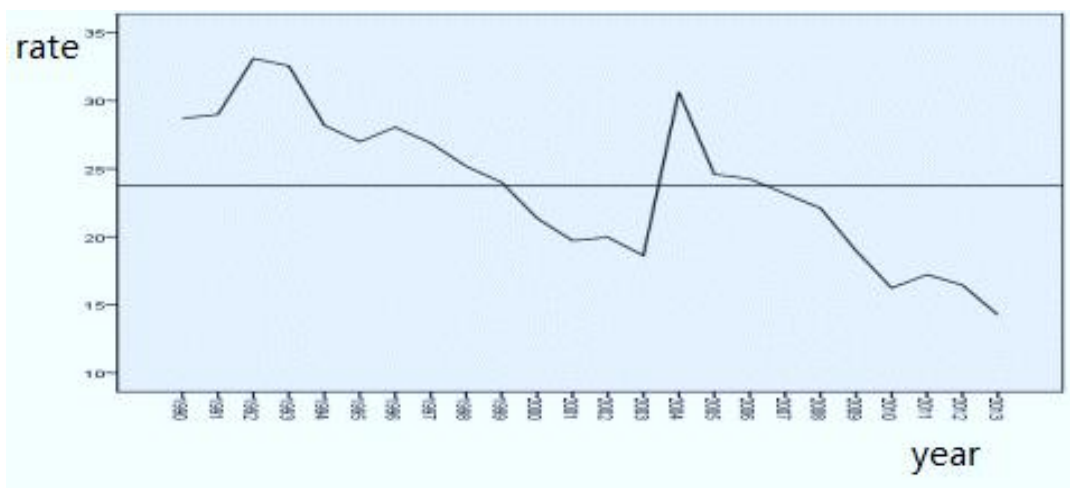

Fig. 1: 1990-2013 incidence of hepatitis B in Jiangsu Province 

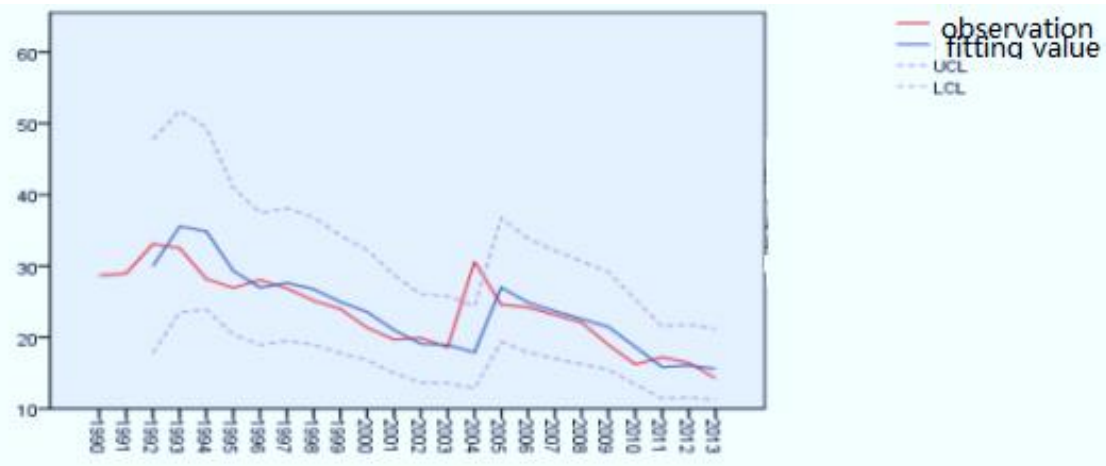

Fig. 2: Model fitting trajectories

Incidence of hepatitis B in Jiangsu has declined in recent years, and prevention measure had positive effect. ARIMA model is applicable in the prediction of hepatitis $\mathrm{B}$ and can provide a basis for short-term forecasting. Control strategy should continue to be consolidated in the future.

\section{Acknowledgements}

This study was funded by the Special funds for basic scientific research business of Southeast University (Grant No.2242017K40040) and the National Natural Science Foundation of China (Project No. 81402769).

\section{Conflict of interest}

The authors declare that there is no conflict of interests.

\section{References}

1. Sehr MA, Joshi KD, Fontanesi JM et al (2017). Markov modeling in hepatitis B screening and linkage to care. Theor Biol Med Model, 14: 11.

2. Chen G, Joan MB, Alison AE et al (2014). Gateway to Care campaign: a public health initiative to reduce the burden of hepatitis $B$ in Haimen City, China. BMC Public Health, 14:754.

3. Heesterbeek H, Anderson RM, Andreasen V, et al (2015). Modeling infectious disease Dynamics in the complex landscape of global health. Science, 347(6227):aaa4339.

4. Osei F B, Duker AA, Stein A (2012). Bayesian structured additive regression modeling of epidemic data: application to cholera. BMC Med Res Methodol, 12:118. 